\title{
Delayed Hemolytic Transfusion Reaction in a Patient with Sickle Cell Disease: Case Report
}

This article was published in the following Dove Press journal:

International Medical Case Reports Journal

\author{
Sawsan A Omer (D) ${ }^{1,2}$ \\ Jafar S Alaesh' \\ Kefah B Algadeeb'
}

'Department of Medicine, King Fahad Hospital Hofuf, Kingdom of Saudi Arabia (KFHH/KSA), Hofuf, Kingdom of Saudi Arabia; ${ }^{2}$ Faculty of Medicine, University of Gezira, Wad Medani, Sudan
Correspondence: Sawsan A Omer Department of Internal Medicine, King Fahad Hospital, Hofuf, Eastern Region, Kingdom of Saudi Arabia

Tel +966553210343

Email sawsanomer63@yahoo.co.uk
Background: Blood transfusion is a key treatment of sickle cell disease (SCD) complications. Delayed hemolytic transfusion reaction (DHTR) is a delayed reaction, that occurs days to weeks following a transfusion, characterized by mild anemia and/or hyperbilirubinemia and is one of the serious complications of blood transfusion. The symptoms of DHTR resemble those of vaso-occlusive crisis secondary to SCD leading to difficulty or delaying in diagnosis of DHTR. DHTR may lead to multiple organ failure and death.

Case Report: A 31-year-old female patient with a known case of SCD presented to our ER in King Fahad hospital Hofuf in the Kingdom of Saudi Arabia, with a history of generalized body ache, exertional dyspnoea, headache and easy fatigability for a few days on a background history of episodic hospital admissions for SCD, but she was admitted 3 times over the previous 6 months and received 6 units of packed red blood cells (PRBCs). The last blood transfusion was 18 days earlier. She was sick and her Hb level was $4.5 \mathrm{~g} / \mathrm{dL}$ with positive Coombs test and positive alloantibodies, diagnosed as DHTR. We treated her with prednisolone tablets $1 \mathrm{mg} / \mathrm{kg}$ daily, intravenous immunoglobulins, $0.4 \mathrm{gm} / \mathrm{kg}$ daily for 5 days, and rituximab $500 \mathrm{mg}$ IV every week for 4 weeks. Her Hb level raised up to $8.2 \mathrm{~g} / \mathrm{dL}$ and she was discharged in good condition.

Conclusion: Identifying risk factors for DHTR by history and presentation is urgently needed in order to risk stratify the transfusion regimen. It is important to avoid additional transfusions in these patients if possible because these may exacerbate the hemolysis and worsen the degree of anemia.

Keywords: sickle cell disease, delayed hemolytic transfusion reaction

\section{Introduction}

Red blood cells (RBCs) transfusion is crucial in the treatment of acute and chronic complications in SCD; however, it is complicated by RBC alloimmunization, iron overload, transfusion reactions and infection. ${ }^{1}$ Transfusion with RBCs matched for $\mathrm{Rh}(\mathrm{D}, \mathrm{C}, \mathrm{E}, \mathrm{c}, \mathrm{e})$ and $\mathrm{K}$ antigens for patients with SCD can be life-saving and is the standard care in many centers but development of antibodies against transfused RBCs may occur leading to DHTR. These alloantibodies are detected by a positive direct antiglobulin test (DAT) and are not always detected in many cases. In DHTR both transfused and autologous RBCs hyperhemolysis occurs and may be accompanied by reticulocytopenia, leading to worsening of the anemia. ${ }^{2}$ SCD patients develop RBCs alloantibodies much more frequently than non-SCD transfused patients, in addition to having a significantly higher risk of suffering from DHTR. $^{3}$ The incidence of alloimmunization in patients with SCD is $5-36 \%$. One complication of alloimmunization is DHTR/H syndrome, with an incidence of $11 \%$. 
In patients with SCD, clinical findings in DHTR occur about 1 week after the RBCs transfusion and include the onset of increased hemolysis associated with pain and severe anemia. ${ }^{4}$ In some studies, the appearance of clinical signs has a median of 9.4 days after the blood transfusion. As a complication of severe intravascular hemolysis, acute chest syndrome, pulmonary hypertension and (multi)organ failure may occur and the overall mortality of DHTR was $6 \%$ in a retrospective study of 99 DHTRs occurring in 69 referral center patients over 12 years. ${ }^{5}$ DHTR can be complicated by hyperhemolysis. ${ }^{6}$

Patients with DHTR often have mixtures of both allo and autoantibodies; rhesus (RH) antibodies and those considered as irregular natural antibodies are frequent. A third of SCD patients will not develop an antibody after a DHTR. ${ }^{7}$ No antibodies are detected in $30 \%$ of patients with DHTR. Prevention is based on the prevention of alloimmunization via the use of matched RBCs for highly immunogenic blood groups, taking into consideration previous transfusion history of the patient especially in patients undergoing occasional transfusion, which is associated with a higher risk of DHTR development than chronic transfusion, in addition to this, use of immunotherapy should be considered. ${ }^{8}$ DHTR represents $4.2 \%$ of all causes of death in SCD, this percentage could be underestimated because DHTR is frequently misdiagnosed as simple vaso-occlusive episodes. Occurrence of DHTR as well as its clinical progression from mild to severe is unpredictable and its prevention in SCD is challenging because only little is known about its mechanism since in some cases of DHTR no antibodies are detected. ${ }^{9}$ Detectable antibodies in SCD DHTR are frequently alloantibodies against antigens such as $\mathrm{Rh}, \mathrm{K}, \mathrm{Fy}, \mathrm{Jk}$, and Ss but antibodies against many other $\mathrm{RBC}$ antigens as well as autoantibodies and nonspecific antibodies can be found. ${ }^{9}$ Risk factors for DHTR include: history of immunization, previous history of DHTR, and transfusion for an acute complication in addition to lower cumulative number of transfused units $(\leq 12$ units $){ }^{9}$ Diagnosis of DHTR in patients with SCD who recently received blood transfusion depends on clinical and laboratory criteria like pain, anemia, urine color, elevated lactate dehydrogenase (LDH) and immune-hematological analysis which may or may not reveal the presence of new antibodies and change in HbA level. ${ }^{9}$ Patients with SCD need close monitoring for detection of DHTR because it could be severe and life threatening so besides supportive measures, specific therapy according to severity criteria should be followed. Severity criteria include: acute chest syndrome, acute pulmonary hypertension, stroke and organ failure (liver or kidney). Intravenous immunoglobulins (IVIg) therapy $(0.4 \mathrm{~g} / \mathrm{kg} /$ day for 3-5 days), is often used for prevention of antibody-mediated immune destruction and its mechanism of action remains incompletely understood. Side effects of IVIg include: hyperviscosity, hypercoagulability, renal toxicity, and hemolysis in non-blood group O. With low $\mathrm{Hb}$ level in DHTR, the risk of hyperviscosity in SCD patients is low. IVIg is recommended as the first-line therapy for patients with DHTR, even in patients with undetectable antibodies because antibodies may develop later. Steroids in high dose is used for immune system modulation and as a first line therapy for severe DHTR with or without IVIg, synergistic action between them has been reported in suppressing macrophage activity. There are no guidelines at present for the use of steroids or IVIg as a first line therapy and no evidence-based studies to support the use of one over the other. Treatment with eculizumab, an inhibitor of the C5-convertase is used early in cases of severe DHTR, with hyperhemolysis to prevent irreversible multi-organ organ failure. Meningococcal vaccine is mandatory with eculizumab treatment. Plasma exchange can also be used in severe cases of hyperhemolysis, it was reported that it can detoxify heme in patients with multiorgan failure without DHTR. In addition to the above therapies blood transfusion may be indicated in the presence of profound anemia and organ hypoxia but rituximab prophylaxis should be given. Use of haptoglobin or hemopexin to detoxify plasma free $\mathrm{Hb}$ and free heme, respectively are promising options which may be explored in the future as therapy for DHTR. ${ }^{9}$ We are presenting DHTR in a 31-year $=$ old female who is known to have SCD with infrequent hospital admissions.

\section{Ethical Considerations}

Approval for the case report was obtained from Hass/King Fahad Hospital research committee and informed written consent was obtained from the patient that the case details could be published.

\section{Case Presentation}

A 31-year-old Saudi female patient with a known case of SCD presented to our ER in King Fahad Hospital, Hofuf in the Kingdom of Saudi Arabia, with a history of generalized body ache, exertional dyspnoea, headache, easy fatigability and dark urine for a few days on a background history of infrequent (episodic) admissions for vasoocclusive crisis but she was admitted 3 times to hospital 
over the previous 6 months because of anemia and thrombocytopenia (secondary to splenomegaly) and she received 6 units of packed red blood cells (PRBCs). Last blood transfusion was 18 days and her hemoglobin $(\mathrm{Hb})$ level before transfusion was $6.5 \mathrm{~g} / \mathrm{dL}$. No history of previous antibodies against RBCs was detected. Prior transfusions blood were matched for $\mathrm{ABO}$ and D antigens only. On examination she was pale and sick. Chest and cardiovascular examination were not remarkable and abdominal examination showed splenomegaly (which was present since last admission). Investigations showed: her $\mathrm{Hb}$ level was $4.5 \mathrm{~g} / \mathrm{dL}$, WBCs: 9.86/L, platelets: 84,000, reticulocytes count $6 \%$, BUN: $3.6 \mathrm{mmol} / \mathrm{L}$, creatinine: 69 mmol/L, AST: $84 \mathrm{U} / \mathrm{L}$, ALT: $20 \mathrm{U} / \mathrm{L}, \mathrm{LDH}: 664 \mathrm{IU} / \mathrm{L}$, total bilirubin: $40.2 \mathrm{mmol} / \mathrm{L}$, direct bilirubin: $14.8 \mathrm{~mol} / \mathrm{L}$, albumin: $40 \mathrm{~g} / \mathrm{L}$, PT: 13, PTT: 30.8, INR:1, with positive direct Coombs test and positive alloantibodies and there was no compatible blood for her for blood transfusion. Hemoglobin electrophoresis: $\mathrm{HbS}$ : 84\%, $\mathrm{HbA} 2: 4 \%, \mathrm{HbF}$ : 12\%, HbA:0\%, G6PD test: normal. HBs Ag, Anti HCV Ab and HIV serology were all negative. The patient was diagnosed to have DHTR and so we treated her with prednisolone tablets $1 \mathrm{mg} / \mathrm{kg}$ daily, intravenous immunoglobulins, $0.4 \mathrm{gm} / \mathrm{kg}$ daily for 5 days, and rituximab $500 \mathrm{mg}$ IV every week for 4 weeks in addition to folic acid $5 \mathrm{mg}$ once per day. The patient did not receive and was not on hydroxycarbamide. Her response to treatment was good and after 1 week of treatment complete blood count (CBC) showed: WBC: 5.63 , and $\mathrm{Hb}: 6.9 \mathrm{~g} / \mathrm{dl}$, platelet: 100,000. The patient was discharged after the second dose of rituximab, in good condition and was re-admitted after 1 week for the third dose of rituximab infusion. $\mathrm{CBC}$ after the 3rd dose of rituximab was as follows: WBC: 6.31/L, $\mathrm{Hb}: 8.2 \mathrm{~g} / \mathrm{dL}$, platelets: 132,000. There was improvement in hemoglobin level in this patient with prednisolone, IVIG and rituximab and without being given a blood transfusion.

\section{Discussion}

Patients with SCD often need RBCs transfusion to save their lives, DHTR is a serious complication of RBC transfusion due to alloimmunization leading to hyperhemolysis and DHTR. ${ }^{10}$ DHTR typically occurs days or weeks following blood transfusion of seemingly compatible RBCs. The delay of onset of DHTR could reflect the recrudescence of alloantibody not detected at the time of the RBC compatibility testing prior to transfusion. Following another blood transfusion, exposure to the implicated alloantigens, the immunological memory generated during the prior transfusion will facilitate an amnestic immune response that results in production of alloantibodies against the transfused units of blood which leads to destruction of the transfused RBC. ${ }^{11}$ According to the guidelines for blood transfusion for patients with SCD, all patients with SCD should receive serologically crossmatched-compatible leukodepleted RBCs and should be monitored with care by doing an antibody screening test including a direct antiglobulin test (DAT). ${ }^{9}$ This is what happened to our patient in this case report where she developed features of DHTR after 18 days of RBC transfusion. Her presentation was with symptoms similar to vaso-occlusive crisis with dropped $\mathrm{Hb}$ and a positive direct Coombs test, positive alloantibodies and her hemoglobin electrophoresis showed zero level of $\mathrm{Hb}$ A only 18 days after a transfusion and this is very specific for DHTR vs other causes of anemia in SCD. The above findings were similar to the study carried out by Habibi et $\mathrm{al}^{5}$ about delayed hemolytic transfusion reaction in adult sickle-cell disease: presentations, outcomes, and treatments of 99 referral center episodes, where they found that the most common clinical manifestations of DHTR were as follows: dark urine/hemoglobinuria in 94\%, painful vaso-occlusive crisis in $89 \%$, and $50 \%$ had a secondary acute chest syndrome. The median $\mathrm{Hb}$ concentration was $5.5 \mathrm{~g} / \mathrm{dL}$ and LDH peak was 1,335 (798-2,086). A total of $61 \%$ of patients had previous antibodies and $28 \%$ had previous DHTRs. ${ }^{5}$ In our patient, her Hb level was $4.5 \mathrm{~g} / \mathrm{dL}$ and LDH was $664 \mathrm{IU} / \mathrm{L}$. In another retrospective study done by Aygun et $\mathrm{al}^{12}$ about the clinical significance of alloimmunization to RBC antigens in sickle cell patients both pediatric and adult sickle cell patients who were followed over a 10 -year period, they found that the alloimmunization rate was $29 \%$ in pediatric and $47 \%$ in adult sickle cell patients when partial or extended RBC antigen match was not performed and DHTR occurred, however it did not result in a severe clinical outcome in most instances. The incidence of alloimmunization in general is high in patients with SCD because of higher prevalence of C, E, Fy ${ }^{\mathrm{a}}, \mathrm{Jk}^{\mathrm{b}}$, and $\mathrm{S}$ polymorphic blood group antigens in donors. Another cause of alloimmunization in SCD may be due to the inflammatory nature of this condition. Additional factors for alloimmunization could be genetic. ${ }^{9}$ The patient in this case report had infrequent hospital admissions for SCD crisis and had occasional transfusions and this put her as a high risk case for the development of DHTR, since, as reported in the literature, patients who had an 
episodic blood transfusion are more prone to develop DHTR than chronic transfusion. A recent prospective study of adults showed that DHTR was exclusively occurring in patients receiving episodic transfusions. ${ }^{9}$ Occasional transfusions are associated with a higher risk of DHTR than chronic transfusion. ${ }^{8}$ Management of DHTR if made early will give the chance for implementation of specific treatments such as steroids, immunoglobulins, erythropoietin and eculizumab with good outcomes. ${ }^{2}$ In one study, supportive care alone was used in $53 \%$, or within 74\% including erythropoietin and sometimes rituximab and/or an immunosuppressant. Additional blood transfusion was either ineffective or worsened hemolysis. ${ }^{5}$ In a case reported by Vlachaki et $\mathrm{al}^{13}$ they had good outcome adjunctive therapy with the use of eculizumab in a patient with sickle cell disease who presented with DHTR with hyperhemolysis. Our patient in this case report received steroids, IVIg, and rituximab infusion and her $\mathrm{Hb}$ level raised without blood transfusion. Prevention of DHTR is based on the attenuation of alloimmunization via extended-matched RBCs or the use of rituximab. ${ }^{2}$ Follow up with antibody screening at regular intervals will help in detection of any possible posttransfusion antibodies which, once known, will help selection of future units for transfusion and thus re-stimulation can be avoided. ${ }^{9}$

\section{Conclusion}

Since DHTR is unpredictable and could be fatal, patient history and clinical presentation is needed to identify risk factors for DHTR. Early diagnosis of DHTR is essential so as to implement specific therapy early and to avoid additional blood transfusions which may exacerbate hemolysis and cause further drops in $\mathrm{Hb}$ levels.

\section{Recommendations}

Improvement of our knowledge regarding presentation and risk factors of DHTR will help in proper management of this life-threatening condition.

Transfusion protocols should be available with strategies to minimize alloimmunization and risk stratification with restricted indications for transfusion for patients with alloimmunization or previous DHTR.

Decision for blood transfusion for SCD patients should be re-evaluated especially for DHTR high risk patients.
Further studies are needed for DHTR patients to highlight optimum ways of management of this life-threatening condition.

\section{Disclosure}

The authors report no conflicts of interest in this work.

\section{References}

1. Fasano RM 1, Meyer EK 2, Branscomb J 3, White MS 4, Gibson RW 5, Eckman JR 6. Impact of red blood cell antigen matching on alloimmunization and transfusion complications in patients with sickle cell disease: a systematic review. Transfus Med Rev. 2019;33 (1):12-23. doi:10.1016/j.tmrv.2018.07.003

2. Pirenne F, Bartolucci P, Habibi A. Management of delayed hemolytic transfusion reaction in sickle cell disease: prevention, diagnosis, treatment. Transfus Clin Biol. 2017;24(3):227-231. doi:10.1016/j. tracli.2017.05.016

3. Fasano RM 1, Miller MJ 2, Chonat S 3, Stowell SR 2. Clinical presentation of delayed hemolytic transfusion reactions and hyperhemolysis in sickle cell disease. Transfus Clin Biol. 2019;26(2):94-98. doi:10.1016/j.tracli.2019.02.002

4. Talano J-AM 1, Hillery CA, Gottschall JL, Baylerian DM, Scott JP. Delayed hemolytic transfusion reaction/hyperhemolysis syndrome in children with sickle cell disease. Pediatrics. 2003;111(6):e661-e665. doi:10.1542/peds.111.6.e661

5. Habibi A, Mekontso-Dessap A, Guillaud C, et al. Delayed hemolytic transfusion reaction in adult sickle-cell disease: presentations, outcomes, and treatments of 99 referral center episodes. Am J Hematol. 2016;91(10):989-994. doi:10.1002/ajh.24460

6. Chonat S 1, Arthur CM 2, Zerra PE 2, et al. Challenges in preventing and treating hemolytic complications associated with red blood cell transfusion. Transfus Clin Biol. 2019;26(2):130-134. doi:10.1016/j. tracli.2019.03.002

7. Thonier $\mathrm{V}$ 1. Immuno-hematological findings in delayed hemolytic transfusion reaction (DHTR). Transfus Clin Biol. 2019;26 (2):102-108. doi:10.1016/j.tracli.2019.02.006

8. Pirenne F 1. Prevention of delayed hemolytic transfusion reaction. Transfus Clin Biol. 2019;26(2):99-101. doi:10.1016/j.tracli.2019. 02.007

9. Pirenne F 1,2, Yazdanbakhsh K 3. How I safely transfuse patients with sickle-cell disease and manage delayed hemolytic transfusion reactions. Blood. 2018;131(25):2773-2781. doi:10.1182/blood-201802-785964

10. Dean CL, Maier L, Chonat SC, et al. Challenges in the treatment and prevention of delayed hemolytic transfusion reactions with hyperhemolysis in sickle cell disease patients. Transfusion. 2019;59 (5):1698-1705. doi:10.1111/TRF.15227

11. Thein SL, Pirenne F, Fasano RM, et al. Hemolytic transfusion reactions in sickle cell disease: underappreciated and potentially fatal. Haematological. 2020;105:539-544. doi:10.3324/haematolo.2019. 224709.

12. Aygun B 1, Padmanabhan S, Paley C, Chandrasekaran V. Clinical significance of RBC alloantibodies and autoantibodies in sickle cell patients who received transfusions. Transfusion. 2002;42(1):37-43. doi:10.1046/j.1537-2995.2002.00007.x

13. Vlachaki E, Gavriilaki E, Kafantari K, et al. Successful outcome of hyperhemolysis in sickle cell disease following multiple lines of treatment: the role of complement inhibition. Hemoglobin. 2018;42(5-6):339-341. doi:10.1080/03630269.20 18.1540353 


\section{Publish your work in this journal}

The International Medical Case Reports Journal is an international, peer-reviewed open-access journal publishing original case reports from all medical specialties. Previously unpublished medical posters are also accepted relating to any area of clinical or preclinical science. Submissions should not normally exceed 2,000 words or 4 published pages including figures, diagrams and references. The manuscript management system is completely online and includes a very quick and fair peer-review system, which is all easy to use. Visit http://www.dovepress.com/testimonials.php to read real quotes from published authors. 